\title{
Study on Th17/Treg Cells and Cytokines in Hashimoto's Thyroiditis with Different lodine Nutrition Status
}

\author{
Cuicui Wang ${ }^{1}$, Zhaoxin $\mathrm{Mu}^{2}$, Yunxia $\mathrm{Chen}^{3}$, Jianfeng Liu ${ }^{3}$, Jinqun $\mathrm{Ma}^{3}$, Chunyan Liu ${ }^{2}$, \\ Zhenjiang Hou ${ }^{2, *}$ \\ ${ }^{1}$ Department of Medical Technology, Cangzhou Medical College, Cangzhou, China \\ ${ }^{2}$ Cangzhou Thyroid Disease Engineering Research Center, Cangzhou Medical College, Cangzhou, China \\ ${ }^{3}$ Endocrinology Department, Cangzhou People's Hospital, Cangzhou, China
}

Email address:

houzhenjiang@sina.com (Zhenjiang Hou)

${ }^{*}$ Corresponding author

\section{To cite this article:}

Cuicui Wang, Zhaoxin Mu, Yunxia Chen, Jianfeng Liu, Jinqun Ma, Chunyan Liu, Zhenjiang Hou. Study on Th17/Treg Cells and Cytokines in Hashimoto's Thyroiditis with Different Iodine Nutrition Status. Science Journal of Public Health. Vol. 9, No. 1, 2021 , pp. 12-22. doi: $10.11648 /$ j.sjph.20210901.12

Received: December 16, 2020; Accepted: January 12, 2021; Published: January 18, 2021

\begin{abstract}
Background: The incidence of AITDs increased with the increase of iodine nutrient levels in the population. HT is one of the most common AITDs. The role of thyroid function stratification, antibody titer, Th17/Treg cells and related factors in the pathogenesis of HT under different iodine nutritional conditions is still unclear. Objective: To investigate the correlation between the thyroid function stratification, autoantibody titer and Th17 cells, Treg cells, cytokines and transcription factors in hashimoto thyroiditis patients with different iodine nutritional status. Methods: Thyroid hormone, autoantibody and urinary iodine levels were measured in 100 hashimoto's thyroiditis (HT) patients and 60 healthy subjects by electrochemical immunoluminescence and iodine-catalyzed arseniummethod. Meanwhile, the proportion and ratio of Th17 cells and Tregs cells in peripheral blood mononuclear cells (PBMC) were determined by immunofluorescence labeling and flow cytometry. The qRT-PCR was used to detect the expression of ROR- $\gamma$ t mRNA and Foxp3 mRNA. Serum IL-17 and TGF- $\beta$ levels were detected by ELISA. Results: Th17 cell proportion, serum IL-17 and ROR- $\gamma \mathrm{t}$ mRNA expression levels in PBMC of HT patients with different iodine nutritional status were all higher than those in the control group $(P<0.05)$, while Tregs cell proportion, serum TGF- $\beta$ and Foxp 3 mRNA level were all lower than those in the control group $(P<0.05)$. Conclusions: The thyroid function, autoantibodies, Th17/Tregs cell proportion, cytokines and transcription factors of HT patients with different iodine nutrition status were changed, hich were involved in the development and progression of HT.
\end{abstract}

Keywords: Th17/Treg Cells, Cytokines, Transcription Factors, Iodine Nutritional Status, Hashimoto's Thyroiditis, Thyroid Autoantibodies

\section{Introduction}

Autoimmune thyroid disease (AITD) is a group of autoimmune diseases (AID) caused by antagonizing thyroid antigens that are exposed to the environment and based on specific genes. Its incidence is gradually increasing, and it has become a common and frequently-occurring disease in the endocrine field. The latest data show that the global prevalence of AITD is about $5 \%$, the prevalence of subclinical AITD is even higher, and women are significantly higher than men. AITD mainly includes the Autoimmune Thyroiditis
(AIT) and Graves' disease (GD) and so on. The former is the main cause of adult hypothyroidism, among which Hashimoto's Thyroiditis (HT) is the most common disease. The prevalence of HT abroad is $1 \%$ to $2 \%$, accounting for about $22.5 \%$ of thyroid diseases. The prevalence of HT in China ranges from $0.3 \%$ to $10 \%$, which can be as high as 22.5 to 40.7 per 100,000 . It can occur in different genders and ages, but mostly 30 to 50 years old, especially in middle-aged women, which accounting for about 90\% [1]. The 10-year change in Universal Salt Iodization (USI) shows that the incidence of AITD increases with the increase of iodine 
nutrition level. AITD is not only the most important cause of thyroid dysfunction, but also can be combined with other AID to form multiple endocrine autoimmune syndrome, etc. It is also closely related to thyroid cancer and seriously endangers human health. AITD can be caused by many factors, such as environmental, genetic and immune disorders, which can lead to specific autoimmune reaction of the thyroid and induce the occurrence of HT [2], diffuse goiter and hyperthyroidism [3]. AITD and the cell subsets of Thl/Th2 have been extensively studied in the past, but the traditional imbalance of Thl and Th2 cells still cannot well explain the pathogenesis of AITD. Recent research has found that Interleukin-17 (IL-17), transforming growthfactor (TGF- $\beta$ ) and retinoic acid-related orphan receptors (ROR- $\gamma \mathrm{t}$ ), Forkhead boxp3 (Foxp3) are the main cytokines and specific transcription factors secreted by Th17 and Treg cells. It plays an important role in immunosuppression and has become the research direction of the occurrence, development and outcome mechanism of immune-related diseases. It plays an important role in immunosuppression and has become the research direction of the occurrence, development and outcome mechanism of immune-related diseases. In recent years, researchers have studied Th17/Treg cells, ROR- $\gamma \mathrm{t}$ and Foxp3 in RA and other AID [4], and found that the proportion of Treg cells may be negatively correlated with the severity of RA, but there is no significant difference from the control group, while Foxp3 is expressed at a low level. The proportion of Treg cells in PBMC of SLE patients was significantly decreased, and the expression of ROR- $\gamma \mathrm{t}$ in urine was up-regulated, but the study results of Treg cell proportion were controversial. There are few studies on Th17/Tregcell ratio and ROR- $\gamma \mathrm{t}$ and Foxp3 expression in AID in China. It was reported that the proportion of Treg cells in PBMC of AITD patients was significantly increased and its inhibitory function was significantly decreased. Immunosuppressive function of Treg cells in HT patients was significantly reduced, but there was no significant difference in cell proportion. Now studies have shown that the proportion of Th17 and/or Treg cells in the pathogenesis of HT is inconsistent. The combined detection of Th17 or Treg cells, ROR- $\gamma$ t, Foxp3, especially the relationship between HT and Th17 has been rarely reported. There are few studies on the relationship between iodine nutrition level and thyroid function and autoantibody level of HT. In particular, studies on iodine nutrition status in thyroid function stratification and antibody titer and the role of Th17/Treg cells and related factors in the pathogenesis of HT have not been carried out. The purpose of this study was to explore the relationship between thyroid function stratification, antibody titer and Th17/Treg cells and related factors in HT with different iodine nutritional status, so as to provide scientific basis for the research on HT precision treatment, prevention and its pathogenesis.

\section{Materials and Methods}

\subsection{Research Objects and Groups}

From September 2019 to August 2020, 100 hospitalized HT patients who received outpatient or initial diagnosis without treatment in Cangzhou People's Hospital Endocrinology were selected as the experimental group, among which 26 were male and 74 were female with an average age of (47.4 \pm 16.8$)$. According to the results of thyroid function examination, HT patients were divided into normal thyroid function group (HT-A group, 31 cases), subclinical hypothyroidism group (HT-B group, 46 cases) and clinical hypothyroidism group (HT-C group, 23 cases). 60 health subjects of the same gender and age were selected as the control group (NC group), including 16 males and 44 females, with an average age of $(45.8 \pm 17.6)$ years old. There were no statistically significant differences in gender and age between the groups. All the patients met the HT diagnostic criteria in Chinese Guidelines for The Diagnosis and Treatment of Thyroid Diseases formulated by Endocrine Society of Chinese Medical Association in 2008 [5], and had not received relevant treatment. Inclusion criteria:(1) Abnormal serum levels of thyroid stimulating hormone (TSH), Anti-thyroid peroxidase antibody and/or anti-thyroglobulin antibody exceeding the upper limit of the normal range; (2) Hard thyroid with or without goiter. (3) Puncture cytology or pathological examination after surgery. Exclusion criteria: (1) The patient has recently suffered from systemic lupus erythematosus, rheumatoid arthritis, asthma, pernicious anemia, multiple sclerosis, systemic sclerosis, etc. (2) Recent ( $\leq 6$ months) viral or bacterial infection medical history; (3) Recent pregnancy ( $\leq 6$ months) or history of childbirth; (4) History of malignant tumor. The assessment of iodine nutritional status is based on the standards revised by the World Health Organization (WHO), the United Nations Children's Fund (UNICEF) and the International Council for the Control of Iodine Deficiency Disorders (ICCIDD) in 2007: Median urinary iodine (MUI) $<100 \mu \mathrm{g} / \mathrm{L}$ is deficiency, $100 \sim 199 \mu \mathrm{g} / \mathrm{L}$ is appropriate, $200 \sim 300 \mu \mathrm{g} / \mathrm{L}$ is excess, and above $300 \mu \mathrm{g} / \mathrm{L}$ is over-dose [6]. This study was approved by the ethics committee, and the study subjects signed an informed consent form.

\subsection{Research Methods and Items}

\subsubsection{Specimen Collection and Processing}

\section{i. Specimen Collection}

All research subjects fasted for more than 12 hours, and collected $10-20 \mathrm{ml}$ of fasting mid-section urine in the morning, placed in a clean polyethylene plastic tube and stored in a refrigerator. $5 \mathrm{~mL}$ of fasting venous blood was collected from 8:00 to 10:00, centrifuged at $3000 \mathrm{rpm} / \mathrm{min}$ for $10 \mathrm{~min}$ after standing at room temperature for 1 hour. Take part of the serum to determine the level of thyroid hormone and autoantibody. Avoid eating high-iodine foods such as kelp and nori and iodine drugs 1 day before urine retention, and avoid increasing the amount of drinking water.

\section{ii. Peripheral Blood Mononuclear Cell (PBMC) Separation}

Separate PBMC by Ficoll density gradient centrifugation, adjust the density of PBMC to $1 \times 10^{6} / \mathrm{L}$, and suspend it in the culture medium of RPMI 1640 with fetal bovine serum. Place 
the PBMC suspension in a 24 -well culture plate, add $25 \mu \mathrm{g} / \mathrm{L}$ of phorbol ester (Alexis, USA) and $1 \mathrm{mg} / \mathrm{L}$ of inomycin (Alexis, USA) to each well, incubate in a $37^{\circ} \mathrm{C}$ incubator for 4 hours, and collect cells.

\subsubsection{Item Detection}

\section{i. Thyroid Function Tests}

Use an immunechemiluminescence instrument (Cobas E601, Roche) to measure serum thyroid stimulating hormone (TSH), total triiodothyronine (TT3), total thyroxine (TT4), free triiodothyronine (FT3), free thyroxine (FT4), anti-thyroid peroxidase antibody (TPOAb) and anti-thyroid globulin antibody ( $\mathrm{TgAb}$ ) with the corresponding reagents (Roche). Due to regional differences, the influence of instruments, reagents and other factors, the reference range of each hospital is different. The normal reference intervals of thyroid hormones and autoantibodies in our hospital were: TSH 0.27 4.2 mIU/L, FT3 2.8 7.1pmol/L, FT412 22pmol $/ \mathrm{L}$, T3 $1.3 \sim 3.10 \mathrm{nmol} / \mathrm{L}$, T466 181nmol/L), TPOAb 0 34IU/mL, TgAb 0 115 IU/mL.

\section{ii. Urine Iodine Determination}

Based on the reaction principle of iodine-catalyzed arsenic-cerium reaction, urine iodine content was detected by as/ce catalytic spectrophotometry (WS/T107-2006) with kit (Wuhan Sentient Beings Biochemical Technology) and instrument (Hitachi, 7600-110).

\section{iii. Proportion of Th17 and Tregs Cells}

Th17 cells were labeled with kits (FITC/CD3, PE/CD4, AlexaFluor647/IL-17, Biolegend, USA). Treg cells were labeled with The Treg cell assay kit (FOXP3AlexaFluor488/CD4PE-CY5/CD25PE, Biolegend, USA). Flow Cytometer (FACSCalibur, BD, USA) was used to conduct experimental operation according to the corresponding instructions. The detection results were expressed as the percentage of positive cells and the fluorescence intensity of positive cells, and the analysis data were obtained by CellQuest software. How to do it: Add $100 \mu \mathrm{L}$ blood sample into flow tube. Add corresponding fluorescent antibody. Incubate in dark for $15 \mathrm{~min}$ at room temperature. Add the red cell lysate. After blending, the mixture was incubated at $4^{\circ} \mathrm{C}$ in dark for $10 \mathrm{~min}$. After adding PBS solution, the mixture was fully mixed and tested on the machine.

\section{iv. The mRNA Expression Levels of ROR-yt and Foxp3}

The mRNA expression levels of ROR- $\gamma \mathrm{t}$ and Foxp3 were detected byqPCR: Total RNA was extracted by Trizol, and cDNA was synthesized with reverse transcription kit (Reverse Transcriptase M-MLV, TaKaRa, D2639A). ROR- $\gamma$ t Forward Primer: 5'-AGTCCAGAACGAGGC AAAGC-3 ', ROR- $\gamma \mathrm{t}$ Reverse Primer: 5' -CTTGGCAAACTCCACCACATA-3 '. Foxp3 Forward Primer: 5'-ATtTTTAGgATtTGAGgTTTTAATA- 3', Foxp3 Reverse Primers: 5 '-CAAAAAAC CCACTAAAAAACTATAC- 3'. qPCR procedure: $95^{\circ} \mathrm{C}, 30 \mathrm{~s} ; 40$ cycles $\left(95^{\circ} \mathrm{C}, 5 \mathrm{~s}, 60^{\circ} \mathrm{C}, 40 \mathrm{~s}\right)$; $95^{\circ} \mathrm{C}, 10 \mathrm{~s} ; 60^{\circ} \mathrm{C}, 60 \mathrm{~s} ; 95^{\circ} \mathrm{C}, 15 \mathrm{~s}$. Each sample was tested for 3 multiple wells, and the data were analyzed by $2-\Delta \Delta \mathrm{CT}$ method.

\section{$v$ IL-17 and TGF- $\beta$ Levels}

Serum IL-17 and TGF- $\beta$ levels were detected by ELISA with the kit. OD450 reading value was taken as the $\mathrm{x}$-coordinate and standard substance concentration was taken as the $y$-coordinate, then the standard curve was prepared, and the levels of IL-17 and TGF- $\beta$ were finally calculated.

\subsubsection{Statistical Methods}

SPSS 20.0 statistical software was used for statistical analysis. Statistical data were presented as rate (\%), $\chi^{2}$ test was used for comparison, normal distribution measurement data were presented as mean \pm standard deviation $(\mathrm{x} \pm \mathrm{s}), \mathrm{P}<$ 0.05 was considered as statistically significant difference in T-test. The measurement data of non-normal distribution are described by median. Kruskal-wallis $\mathrm{H}$ test was used for comparison between groups, and Spearman was used for single-factor correlation analysis. $\mathrm{P}<0.05$ was considered as statistically significant difference.

\section{Results}

\subsection{Comparison of Thyroid Hormone and Autoantibody Levels in Different HT Patients}

The results showed that the serum TSH level of 100 HT patients was significantly higher than that of NC patients, and gradually decreased with the thyroid function. The TSH level of HT-A, HT-B and HT-C groups increased successively, with the most significant increase in HT-C group. Pair comparison between the three groups showed statistically significant differences $(\mathrm{P}<0.05)$. The serum levels of FT3 and FT4 in HT and HT groups were all lower than that in NC group, and showed a decreasing trend with the gradual decline of thyroid function among the three groups. HT and HT-C were statistically different from the NC group $(\mathrm{P}<0.05)$. The differences between HT-A, HT-B and NC groups were not statistically significant $(\mathrm{P}>0.05)$. The serum T3 and T4 levels of HT and HT three groups were lower than those of $\mathrm{NC}$, but there was no statistically significant difference between the groups $(\mathrm{P}>0.05)$. The serum TPOAb and TgAb levels were higher than that of $\mathrm{NC}$ $(\mathrm{P}<0.01)$. With the aggravation of the disease, the serum TPOAb and TgAb levels of HT-A, HT-B and HT-C groups increased successively, and there were significant differences among each group $(\mathrm{P}<0.01)$. Table 1 .

\subsection{Comparison of Th17 and Tregs Cells and Their Cytokines and Transcription Factors}

Thisstudy found IL-17 cells proportion, ratio of Th17/Treg, IL-17, TGF- $\beta$ and ROR- $\gamma$ twere higher in HT patients than that in NC group, and increased gradually. There were statistically significant differences among the three groups $(\mathrm{P}<0.05$ or $<0.01)$. The proportion of Treg cells and Foxp3 levels were significantly lower than those of the control group, and decreased gradually from HT-A to HT-C. The differences between HT-C, HT-B and HT-A groups were statistically significant $(\mathrm{P}<0.05)$. Table 2 . 
Table 1. Comparison of Thyroid Hormone and Autoantibody Levels in Different HT Patients.

\begin{tabular}{|c|c|c|c|c|c|c|c|c|}
\hline Group & Cases & TSH (mIU/L) & FT3 (pmol/L) & FT4 $(\mathrm{pmol} / \mathrm{L})$ & T3 (nmol/L) & T4 (nmol/L) & TPOAb IU/ml & TgAb IU/ml \\
\hline HT & 100 & $5.94 \pm 1.61^{\boldsymbol{\Lambda}}$ & $4.08 \pm 1.28 *$ & $18.6 \pm 11.37 *$ & $1.79 \pm 0.38$ & $105.49 \pm 19.81$ & $541.2 \pm 236.3^{\mathbf{\Lambda}}$ & $151.32 \pm 68.7^{\mathbf{\Lambda}}$ \\
\hline HT-A & 31 & $8.92 \pm 1.67 *$ & $3.82 \pm 0.98^{*}$ & $18.02 \pm 8.02 *$ & $1.68 \pm 0.41$ & $106.32 \pm 20.94$ & $241.8 \pm 162.4^{\boldsymbol{\Lambda}}$ & $67.39 \pm 1.27^{\mathbf{\Lambda}}$ \\
\hline HT-B & 46 & $11.02 \pm 1.35^{*}$ & $3.09 \pm 1.04 *$ & $17.36 \pm 7.93 *$ & $1.59 \pm 0.52$ & $104.96 \pm 21.62$ & $392.7 \pm 183.2^{\boldsymbol{\Lambda}}$ & $189.16 \pm 38.7^{\mathbf{\Lambda}}$ \\
\hline HT-C & 23 & $18.09 \pm 1.68^{*}$ & $2.83 \pm 1.13^{*}$ & $15.02 \pm 7.36^{*}$ & $1.62 \pm 0.61$ & $103.67 \pm 18.69$ & $761.4 \pm 213.9^{\Delta}$ & $316.3 \pm 127.7^{\mathbf{\Lambda}}$ \\
\hline $\mathrm{NC}$ & 60 & $2.46 \pm 1.23$ & $4.91 \pm 0.58$ & $19.14 \pm 7.09$ & $1.82 \pm 0.42$ & $104.9 \pm 20.16$ & $9.16 \pm 1.02$ & $15.68 \pm 0.97$ \\
\hline
\end{tabular}

Note: Comparison with control group, ${ }^{*} P<0.05,{ }^{\mathbf{\Lambda}} \mathrm{P}<0.01$.

Table 2. Comparison of Th17 and Tregs Cells and Their Cytokines and Transcription Factors.

\begin{tabular}{|c|c|c|c|c|c|c|c|c|}
\hline Group & Cases & Th17 cell\% & Tregcell\% & Th17/Treg & IL-17 (pg/ml) & TGF- $\beta(\mathrm{pg} / \mathrm{ml})$ & ROR- $\gamma \mathrm{t}$ & Foxp3 \\
\hline HT & 100 & $1.72 \pm 0.47 *$ & $2.47 \pm 0.38^{*}$ & $0.92 \pm 041^{\boldsymbol{\Lambda}}$ & $34.9 \pm 7.28^{\mathbf{\Lambda}}$ & $26.36 \pm 2.04 *$ & $7.12 \pm 1.29^{*}$ & $0.74 \pm 0.21^{*}$ \\
\hline HT-A & 31 & $1.26 \pm 0.24$ & $5.62 \pm 0.98$ & $0.21 \pm 0.06$ & $14.62 \pm 2.41$ & $17.32 \pm 3.94$ & $1.67 \pm 0.46$ & $1.92 \pm 0.57$ \\
\hline HT-B & 46 & $1.69 \pm 0.31 *$ & $3.09 \pm 1.04 *$ & $0.66 \pm 0.08^{\boldsymbol{\Lambda}}$ & $22.9 \pm 3.12 *$ & $21.96 \pm 4.28 *$ & $3.87 \pm 0.32 *$ & $1.39 \pm 0.64 *$ \\
\hline HT-C & 23 & $2.17 \pm 0.27^{*}$ & $2.16 \pm 1.13^{*}$ & $1.24 \pm 0.02^{\mathbf{\Delta}}$ & $37.3 \pm 6.94^{\mathbf{\Lambda}}$ & $31.67 \pm 3.91 *$ & $8.17 \pm 0.41^{*}$ & $0.58 \pm 0.51 *$ \\
\hline $\mathrm{NC}$ & 60 & $0.91 \pm 0.28$ & $6.73 \pm 0.97$ & $0.12 \pm 0.04$ & $13.17 \pm 2.84$ & $15.97 \pm 3.68$ & $1.49 \pm 0.38$ & $1.89 \pm 0.63$ \\
\hline
\end{tabular}

Note: Comparison with control group, ${ }^{*} P<0.05,{ }^{\mathbf{\Lambda}} \mathrm{P}<0.01$.

\subsection{Comparison of Th17 and TregsCells and Their Cytokines and Transcription Factors in HT Patients with Different Urinary Iodine Levels}

The changes of Th17 and Tregs cells and their cytokines and transcription factors were basically consistent with those of HT patients with different iodine nutritional status such as iodine deficiency, iodine amount, iodine excess and iodine much excess. Among them, the decrease of Treg cell ratio, Th17/Treg ratio and the increase of IL-17 were significant, but there were no statistically significant differences between the groups. Table 3 .

Table 3. Comparison of Th17 and TregsCells and Their Cytokines and Transcription Factors in HT Patients with Different Urinary Iodine Levels.

\begin{tabular}{|c|c|c|c|c|c|c|c|c|}
\hline Group & Cases & Th17 cell\% & Tregcell $\%$ & Th17/Treg & IL-17 (pg/ml) & TGF-- $\beta$ & ROR- $\gamma \mathrm{t}$ & Foxp3 \\
\hline deficiency & 9 & $1.69 \pm 0.38$ & $2.54 \pm 0.36$ & $0.86 \pm 0.43$ & $31.87 \pm 6.93$ & $13.9 \pm 2.18$ & $6.94 \pm 1.82$ & $0.8 \pm 0.19$ \\
\hline adequate & 46 & $1.74 \pm 0.46$ & $2.52 \pm 0.28$ & $0.95 \pm 0.38$ & $32.07 \pm 7.32$ & $14.2 \pm 2.36$ & $7.18 \pm 1.43$ & $0.7 \pm 0.23$ \\
\hline excess & 32 & $1.89 \pm 0.51$ & $2.32 \pm 0.33$ & $1.28 \pm 0.47$ & $33.24 \pm 6.87$ & $15.0 \pm 2.27$ & $7.93 \pm 1.58$ & $0.7 \pm 0.20$ \\
\hline
\end{tabular}

\subsection{Comparison of Th17 and Tregs Cells and Their Cytokines and Transcription Factors in HT Patients with Different TPOAb and $\mathrm{Tg} A b$ Levels}

Among the 100 HT patients, the number of TPOAb and $\mathrm{TgAb}$ antibody-positive cases were 91 and 84 with a median of $391.27 \mathrm{IU} / \mathrm{mL}$ and $182.59 \mathrm{IU} / \mathrm{mL}$ respectively. Using this data as a critical value, HT patients was divided into high TPOAb group, low TPOAbgroup, high TgAb group, and low
TgAb group. The test results showed that the levels of Tregs and Foxp3 in the low TPOAb and TgAb groups were higher than those in the high TPOAb and TgAb groups, while the Th17 and Tregs cells and their cytokines and transcription factors in the high TPOAb and TgAb groups were higher than those in the low TPOAb and TgAb groups. Among them, the results of Th17/Treg, IL-17 and ROR- $\gamma$ t were more obvious, but the differences were not statistically significant. Table 4.

Table 4. Comparison of Th17 and Tregs Cells and Cytokines and Transcription Factors in HT Patients with Different TPOAb and TgAb Levels.

\begin{tabular}{|c|c|c|c|c|c|c|c|}
\hline Group & Th17 cell\% & Tregcell\% & Th17/Treg & IL-17 (pg/ml) & TGF- $\beta$ & ROR- $\gamma \mathrm{t}$ & Foxp3 \\
\hline High TPOAb & $2.16 \pm 0.48$ & $2.62 \pm 0.29$ & $1.36 \pm 0.63$ & $39.8 \pm 7.81$ & $27.53 \pm 2.31$ & $7.73 \pm 1.42$ & $0.67 \pm 0.23$ \\
\hline Low TPOAb & $1.81 \pm 0.43$ & $3.02 \pm 0.35$ & $1.21 \pm 0.59$ & $34.2 \pm 6.95$ & $25.31 \pm 2.19$ & $7.21 \pm 1.36$ & $0.82 \pm 0.19$ \\
\hline High TgAb & $1.83 \pm 0.39$ & $2.35 \pm 0.41$ & $1.12 \pm 0.67$ & $36.9 \pm 7.84$ & $26.47 \pm 1.90$ & $8.12 \pm 1.37$ & $0.61 \pm 0.18$ \\
\hline Low $\mathrm{TgAb}$ & $1.65 \pm 0.51$ & $2.64 \pm 0.25$ & $0.83 \pm 0.41$ & $32.7 \pm 7.36$ & $24.68 \pm 2.26$ & $7.06 \pm 1.25$ & $0.78 \pm 0.20$ \\
\hline HT & $1.72 \pm 0.47$ & $2.47 \pm 0.38$ & $0.92 \pm 0.41$ & $34.9 \pm 7.28$ & $26.36 \pm 2.04$ & $7.12 \pm 1.29$ & $0.74 \pm 0.21$ \\
\hline
\end{tabular}

\subsection{Correlation Between Th17/Treg Ratio and Related Factors and Thyroid Autoantibodies}

Th17 cell proportion and Th17/Treg ratio were positively correlated with Th17, TGF- $\beta$, ROR- $\gamma \mathrm{t}(\mathrm{P}<0.05)$, and negatively correlated with Foxp3 $(\mathrm{P}<0.05)$. The ratio of Treg cells was negatively correlated with Th17/Treg ratio, Th17 cell ratio, TGF- $\beta$, ROR- $\gamma \mathrm{t}(\mathrm{P}<0.05)$, and positively correlated with Foxp3.
The Th17/Treg ratio was significantly positively correlated with serum TPOAb and TgAb titers $(\mathrm{P}<0.05$ and $\mathrm{P}<0.01)$.

\section{Discussion}

HT is a kind of chronic inflammatory AIT that takes self-thyroid tissue as the antigen, also known as chronic lymphocytic thyroiditis (CLT). It is a common form of AIT 
and the most common type of AITD. HT patients are mainly characterized by TPOAb, TgAb and thyroid lymphocyte infiltration, which eventually lead to thyroid tissue destruction, which is the most common cause of hypothyroidism. Abnormal iodine intake is also an important factor in inducing AITD, but the results of studies on thyroid hormone, autoantibodies and urine iodine levels in HT patients are not the same. The titers of TgAb and TPOAb in HT and hypothyroidism groups were significantly higher than those in the control group, and they were higher in the GD group than in the HT group, while the TSH content was not statistically different from the control group [7]. TSH in HT patients was significantly higher than that in the control group, $\operatorname{TgAb}$ and TPOAb titers were significantly higher than in GD patients and the control group, and TPOAb in GD patients was lower than that in HT patients. The difference was statistically significant, while the difference in $\mathrm{TgAb}$ between the two groups was not statistically significant. There was no statistical difference in the level of FT3 and FT4 between HT patients and the control group [8]. Serum TgAb and TPOAb titers of HT and GD patients were significantly higher than those of the control group, and the HT group was higher than the GD group with statistically significant difference [9]. The titers of TPOAb and TgAb in HT and GD patients were higher than those in the control group without statistically significant difference [10]. It reported that serum $\mathrm{TSH}, \mathrm{TgAb}$, and TPOAb levels in HT patients were significantly higher than those in the control group, while serum FT4 levels were lower $(\mathrm{P}<0.05)$. Serum FT3 levels were similar between the hypothyroidism group and the normal thyroid function group without statistically significant difference $(\mathrm{P}>0.05)$. The serum TSH and TPOAb levels in the hypothyroidism group were higher than those in the normal thyroid function group $(\mathrm{P}<0.05, \mathrm{P}<0.01)$, but the serum FT4 levels were lower $(\mathrm{P}<0.05)$. There was no significant difference in serum FT3 and $\mathrm{TgAb}$ titers between hypothyroidism and normal thyroid function groups $(\mathrm{P}>0.05)$ [11]. The levels of FT3 and FT4 in HT patients were similar with that in control group, but the levels of TSH, TPOAb, TgAb and MUI were significantly higher in HT patients [12]. Ma QQ believed that the serum TSH levels, TPOAb, and TgAb titers of HT patients were significantly higher than those of the control group $(\mathrm{P}<0.01)$, while the serum FT4 level was significantly lower, and the serum FT3 level was similar [13]. ChenYM reported that there was no statistically significant difference between TSH, FT3, FT4 in HT patients and the control group, but the titers of $\mathrm{TPOAb}$ and $\mathrm{TgAb}$ were significantly higher than those in the control group $(\mathrm{P}<0.05)$ [14]. The above research results show that the titers of $\mathrm{TgAb}$ and TPOAb in HT patients are significantly higher than those in the control group, but the changes in thyroid function indicators (TSH, FT3, FT4) and antibody titers levels between the HT and GD groups are inconsistent. This may be related to the disease composition ratio, severity and stage.

Our research results show that the serum TSH level of HT patients is significantly higher than that of $\mathrm{NC}$, and the TSH levels of HT-A, HT-B, and HT-C groups increase sequentially with the gradual decline of thyroid function, among which HT-C increases the most. The differences were statistically significant $(\mathrm{P}<0.05)$. The serum FT3 and FT4 levels showed a decreasing trend, the differences between HT and HT-C and NC were statistically significant $(\mathrm{P}<0.05)$, while the differences of HT-A, HT-B and NC was not statistically significant $(\mathrm{P}>0.05)$. Serum T3 and T4 levels of HT patients or other group patients were lower than those of $\mathrm{NC}$ without statistically significant difference $(\mathrm{P}>0.05)$. The titers of $\mathrm{TPOAb}$ and $\mathrm{TgAb}$ were higher than those of $\mathrm{NC}(\mathrm{P}<0.01)$. With the worsening of the disease, the titers of serum TPOAb and TgAb in the HT-A, HT-B, and HT-C groups increased successively, and the differences between the groups were statistically significant $(\mathrm{P}<0.05)$. With the increase of urine iodine level in HT patients, TSH, TPOAb, and TgAb increased, while serum FT3, FT4, TT3, and TT4 decreased. With the progression of the disease, the serum TPOAb and $\mathrm{TgAb}$ titers increased sequentially, and there were significant differences between the groups $(\mathrm{P}<0.05)$. Serum TSH levels in the high TPOAb group and high TgAb group were higher than those in the low TPOAb group, low TgAb group, and control group. The levels of FT3 and FT4 were lower than those in the low TPOAb and TgAb groups. The FT3 and FT4 levels were lower than the low TPOAb and TgAb groups. However, there was no statistically significant difference in T3 and T4 levels between the low TgAb FT3 and the control group, high TPOAb group, high TgAb group, low TPOAb group, and low $\mathrm{TgAb}$ group. The results of the study showed that MUI is closely related to thyroid disease, and there is a certain correlation between thyroid function and urinary iodine level and thyroid autoantibody titer in AITD patients. The urine iodine level $(491.20 \mu \mathrm{g} / \mathrm{L})$ of HT patients was significantly higher than that of the control group $(256.50 \mu \mathrm{g} / \mathrm{L})$, and the positive rates of $\mathrm{TgAb}, \mathrm{TPOAb}$ and antibody titers in the high iodine urine HT group were higher than those in the low urine iodine HT group [15], The difference was statistically significant $(\mathrm{P}<0.05)$, suggesting that excess iodine significantly increased the autoantibody titers of AITD patients.

Most studies believe that ITD is the result of a combination of environmental, genetic, and immune imbalances, among which immune factors are recognized as the main cause of disease. Th17/Treg is a pair of Th cell subgroups with pro-inflammatory/anti-inflammatory effects, with independent differentiation and regulation mechanisms, and mutual inhibition. Thl7/Treg has been confirmed to play an important role in the pathogenesis of AITD [16]. Th17 cells secrete high levels of IL-17, so that pro-inflammatory factors induce the production of a variety of pro-inflammatory cytokines and chemokines, and then participate in the body's inflammatory response and immune defense [16, 17]. Treg cells secrete cytokines and direct contact to prevent the activation of self-reactive $\mathrm{T}$ cells, exert an immunosuppressive effect and then inhibit the occurrence of AID. It can also regulate the activation process of effector $T$ cells at the transcriptional level through Foxp3. Previous studies have shown that Th17 $\left(\mathrm{CD} 4^{+} \mathrm{IL}-17^{+}\right)$cells and $\mathrm{T}$ 
regulatory cells $\left(\mathrm{CD}^{+} \mathrm{CD} 25^{+}(\mathrm{H}) \mathrm{Foxp}^{+}\right)$play a role in the induction of AID. In recent years, studies have found that there is an imbalance of Th17/Treg cells in AITD patients, and there is a certain correlation between cytokines and thyroid autoantibodies, but the results of the studies are not the same.

Research on Th17 cells and their factors in HT, IL-17 was strongly expressed in the thyroid tissue of HT patients, while IL-17 was almost not expressed in GD patients and the control group. The level of IL-17mRNA in PBMC is elevated, while it is lower in GD patients and normal people [18]. The levels of IL-17 in the serum of HT and GD patients were significantly higher than those of the control group, especially HT. It shows that Th17 cells in HT patients have stronger ability to produce IL-17. Some studies have found that Th17 cells, not Th1 cells, play a key role in the pathogenesis of HT [19], and the proportion of Th17 cells in PBMC increases [20]. The proportion of Th17 cells in PBMC in AITD patients increased significantly, the increase was more obvious in Hashimoto's hypothyroidism patients. That suggested the increase of Th17 cell proportion may be closely related to Hashimoto's hypothyroidism [21]. The levels of IL-17 mRNA and serum IL-17 in the thyroid tissue of HT patients were significantly higher than those of the control group, especially it was higher in the HT hypothyroidism group than in the HT normal thyroid function group. While the Foxp3 mRNA expression and IL-10 levels were significantly lower, but there is no significant difference between HT hypothyroidism group and normal thyroid function group [22]. It is suggested that Th17 cells in thyroid tissue and PBMC of HT patients have an enhanced response, and Th17 cells may be involved in the occurrence and development of HT. Chen ZJreported that the proportion of Th17 cells, IL-17 and TGF- $\beta$ levels in HT patients were significantly higher than those in the control group, and the proportion of Th17 cells and IL-17 levels were positively correlated with TPOAb and TgAb titers [23]. The ratio of Th17/T $\left(\mathrm{CD}^{+}\right)$in PBMC of HT patients increased significantly and positively correlated with $\mathrm{TgAb}$ titers, but not correlated with TPOAb titers [24]. Sun QK reported that the IL-6 concentration, CD4 ${ }^{+} \mathrm{IL}-17 \mathrm{~A}^{+} \mathrm{T}$ cell ratio, Th17/Treg cell ratio and ROR- $\gamma$ t expression level in HT patients were significantly higher than those in the control group $(\mathrm{P}<0.05)$, while $\mathrm{CD} 4{ }^{+} \mathrm{CD} 25^{+} \mathrm{Foxp}^{+} \mathrm{T}$ cell ratio, TGF- $\beta$ concentration and Foxp3 expression level were significantly reduced $(\mathrm{P}<0.05)$, and the ratio of IL-6/TGF- $\beta$ and Th17/Treg was positively correlated $(\mathrm{P}<0.05)$. After TGF- $\beta$ stimulation of $\mathrm{CD}^{+} \mathrm{T}$ cells in normal human peripheral blood, the proportion of Treg cells increased significantly, while the proportion of Th17 cells and the ratio of Th17/Treg decreased significantly $(\mathrm{P}<0.05)$. He believed that TGF- $\beta$ signal abnormality may be one of the factors leading to the imbalance of Th17/Treg cells in HT patients [25]. XueHB found that the Th17 cell proportion, Th17/Treg ratio, serum IL-17 and ROR- $\gamma \mathrm{t}$ mRNA levels in the PBMC of HT patients were significantly higher than those in the NC group, while the Treg cell proportion and Foxp3 mRNA levels were clearly lower $(\mathrm{P}<0.01)$. The changes were more obvious with the progress of different stages of HT. Th17/Treg ratio was clearly positively correlated with serum TPOAb and TgAb titers, while the serum TGF- $\beta$ was slightly lower than that of the $\mathrm{NC}$ group, and the difference was not statistically significant $(\mathrm{P}>0.05)$ [26]. Jin $\mathrm{X}$ reported that Th17/Treg ratio, ROR- $\gamma \mathrm{t}$ mRNA level and serum IL-17 in PBMC of HT patients were significantly higher than those in the control $(\mathrm{P}<0.05)$, but the proportion of Treg cells and Foxp3 mRNA levels were significantly lower $(\mathrm{P}<0.05)$, the serum TGF- $\beta$ was slightly lower. The difference was not statistically significant $(\mathrm{P}>0.05)$ [27]. Chen YM found that the proportion of Th17 cells in the PBMC of HT patients was higher than that in the control without statistically significant ( $\mathrm{P}>0.05$ ), but the ratio of Treg/Th17 was significantly lower. Whilethe difference between the HT patients with normal thyroid function and the control was not statistically significant. TPOAb and $\mathrm{TgAb}$ titers were not significantly correlated with the proportion of Treg and Th17 cells or the ratio of Treg/Th17 $(\mathrm{P}>0.05)$ [14]. Other Th17 cytokine levels are altered in Hashimoto's thyroiditis [28].

Our results show that as the condition of HT patients worsened, the proportion of Th17 cells, IL-17 and ROR- $\gamma \mathrm{t}$ expression levels gradually increased, while the proportion of Treg cells and Foxp3 mRNA expression gradually decreased accompanied by obvious dynamic changes. Patients with hypothyroidism have more obvious changes than patients with subclinical hypothyroidism and HT patients with normal thyroid function. This is basically consistent with the results of Xue HB [26]. This shows that Th17/Treg imbalance is dynamically changing in patients with different degrees of HT and is closely related to thyroid autoantibodies. It also shows that Th17/Treg imbalance may promote the production of thyroid autoantibodies. It not only participates in the pathogenesis of HT, but also reflects changes in the disease, and participates in and aggravates the autoimmune damage of HT patients. The change in Th17/Treg ratio may be caused by changes in thyroid function. It is unclear whether it is directly related to the immune response of HT patients.

Most of the above research results show that Th17 cell proportion, Th17/Treg ratio, ROR- $\gamma \mathrm{t}$ mRNA level and serum IL-17 level in HT patients are positively correlated with TPOAb and TgAb titers. But in some research Th17 cell ratio is higher than those in the control group without statistical significance $(\mathrm{P}>0.05)$, there is no significant correlation between Th17 cell proportion, Treg/Th17 ratio and TPOAb and $\mathrm{TgAb}$ titers, serum TGF- $\beta$ level is lower or significantly higher than the control group.

Marazuela reported that the proportion of thyroid tissue and PBMC in AITD patients was significantly higher than that in the control group. Treg cells have functional defects, their inhibitory ability is significantly reduced, and they cannot effectively down-regulate the body's immune response to prevent the occurrence of AITD [29].

Gao ST found that the number of CD4 ${ }^{+} \mathrm{T}$ cells in the PBMC of $40 \mathrm{HT}$ patients was not statistically different from that of the control group $(\mathrm{P}>0.05)$, while the proportion of $\mathrm{CD} 4{ }^{+} \mathrm{CD} 25^{+}$Tregs, TGF- $\beta 1$ and Foxp3 mRNA levels significantly reduced. The ratio of $\mathrm{CD} 4^{+} \mathrm{CD} 25^{+}$Tregs $/ \mathrm{CD} 4^{+} \mathrm{T}$ cells, the proliferation inhibition rate of $\mathrm{CD} 4{ }^{+} \mathrm{CD} 25^{+}$Tregs on 
$\mathrm{CD} 4^{+} \mathrm{CD} 25^{-} \mathrm{T}$ cell and the levels of TGF- $\beta 1$ mRNA and Foxp3 mRNA were all clearly lower. This indicates that the proportion and function of $\mathrm{CD} 4^{+} \mathrm{CD} 25^{+}$Treg cells in PBMC are significantly reduced [30]. It suggested that the decrease in the proportion of $\mathrm{CD} 4{ }^{+} \mathrm{CD} 25^{+}$Tregs and/or dysfunction and the decrease of Foxp 3 and TGF- $\beta 1$ may be involved in the occurrence and development of HT. Mao $\mathrm{C}$ reported that the ratio of $\mathrm{CD}^{+} \mathrm{CD} 25^{+} \mathrm{Foxp}^{+}{ }^{+}$Treg cells $/ \mathrm{CD} 4^{+} \mathrm{T}$ cells in the PBMC of AITD patients was significantly lower than that of HT patients with normal thyroid functionn especially Foxp $3{ }^{+} \mathrm{CD} 4{ }^{+} \mathrm{CD} 25^{+}$Treg cells, while the proportion of activated $\mathrm{CD}^{+} \mathrm{T}$ cells increased [31]. Zheng LT found that the proportion of $\mathrm{CD} 4^{+} \mathrm{CD} 25^{+}$Treg cells and Foxp 3 mRNA levels in PBMC of $50 \mathrm{HT}$ patients were significantly lower than those of the control group ( $\mathrm{P}<0.05)$, while the serum TGF- $\beta 1$ level was higher $(\mathrm{P}<0.05)$ [32]. Yang $\mathrm{ZF}$ reported that the ratio of Treg/CD4 ${ }^{+} \mathrm{T}$ cells and the expression level of Foxp3 mRNA of $\mathrm{CD}^{+} \mathrm{T}$ cells in HT patients were significantly reduced, but there was no statistical difference between hypothyroidism, subclinical hypothyroidism and normal thyroid function groups $(\mathrm{P}>0.05)$. Pearson correlation analysis showed that the proportion of Treg cells was negatively correlated with the patient's age and TPOAb titer $(\mathrm{P}<0.05)$, but had nothing to do with thyroid function [33], suggesting that the proportion of Treg cells was significantly reduced and accompanied by immune function defects. Jin X reported that the proportion of Treg cells and the level of Foxp3 mRNA were lower than those of the control group $(\mathrm{P}<0.05)$ [27]. $\mathrm{Bi}$ JHfound that the ratio of $\mathrm{CD} 4^{+} \mathrm{CD} 25^{+} \mathrm{Foxp} 3^{+}$Treg/CD $4^{+} \mathrm{T}$ cells, the ratio of $\mathrm{CD} 4^{+} \mathrm{CD} 25^{+}$Foxp $3{ }^{+}$Treg/CD $4{ }^{+} \mathrm{CD} 25^{+}$Treg and IL-10 levels were significantly lower than the control group $(\mathrm{P}<0.05)$ [34]. Chen YMreported that the proportion of Treg cells was significantly lower than that of the control group, while the proportion of Th17 cells was higher, but the difference was not statistically significant $(\mathrm{P}>0.05)$. The ratio of Treg/Th17 was significantly lower, but there was no statistical difference between the HT patients with normal thyroid function and the control group [14]. ZouGHfound that the proportion of Treg cells in PBMC of HT patients was lower than that in the control group $(\mathrm{P}<0.05)$ and the proportion of Treg cells in normal or hypothyroid patients was lower. While the difference between serum FT3 and TgAb levels was not statistically significant $(\mathrm{P}>0.05)$. TPOAb titers are negatively correlated with the proportion of Treg cells, while $\mathrm{TgAb}$ titers have no correlation with the proportion of Treg cells [11]. It suggests that the decrease in the proportion of Treg cells may be involved in the pathogenesis of HT. Glick found that $\mathrm{Treg} / \mathrm{CD} 4^{+} \mathrm{T}$ cells were not significantly different from the control group, but the inhibitory ability was reduced [35], suggesting that the function of Treg cells in AITD patients was reduced. The $\mathrm{T}$ cells expressing Foxp3 in the PBMC of AITD patients increase, but their ability to inhibit cell proliferation decreases. Most of them cannot down-regulate autoimmune response and reduce the damage of inflammation to thyroid tissue. This shows that Treg cells of AITD patients have inhibitory defects in autoimmune response [36]. The above research results show that the dysfunction of Treg cells in the development of AITD is more important than the change in the proportion of Treg cells alone, it can reduce the body's ability to suppress the immune response and lead to the occurrence of AITD. The immunosuppressive ability of Treg cells in the thyroid tissue and PBMC of HT patients decreases, while the immune response ability of Th17 cells is enhanced. Treg/Th17 immune imbalance may be involved in the occurrence and development of HT.

Our research results show that the proportion of Treg cells, Th17/Treg ratio and Foxp3 mRNA expression levels in HT patients show a progressive decline with different stages of disease progression. In summary, the results of the above studies indicate that the decrease in the proportion of Treg cells accompanied by functional abnormalities in HT patients is common. While there are also some reports that the proportion of Treg cells increases or does not change significantly.

HT is a common organ-specific AITD, and its pathogenesis has always been a hot research topic. Previous studies have suggested that organ-specific AID is mainly related to Th1 cells. With the in-depth study of T cell subpopulations, the understanding of autoimmunity has changed significantly [37]. Th17 and Treg cells are $\mathrm{CD}^{+}{ }^{+} \mathrm{T}$ cell subsets with opposite functions. Th17 and Treg cells are $\mathrm{CD}^{+} \mathrm{T}$ cell subsets with opposite functions. The former mainly mediates inflammation by secreting high levels of IL-17 and other cytokines. The latter mainly exerts immunosuppressive function through direct contact between cells and secretion of inhibitory cytokines (TGF- $\beta$, IL-10, etc.). Both and their cytokines play an important role in the pathogenesis of AID [38]. Recent studies have confirmed that Th17, Treg cells and their cytokines are related to the occurrence of a variety of AIDs. The increase in the proportion of Th17 cells and the decrease in the proportion of Treg cells in the PBMC of HT hypothyroidism patients are more obvious than those in patients with normal thyroid function and subclinical hypothyroidism [26], That indicated that abnormal thyroid function may affect Th17 or Treg cell function.

Our research results showed that the Th17 cell proportion, Th17/Treg ratio, serum IL-17 and ROR- $\gamma$ t in PBMC of HT patients were significantly increased, while the Treg cell proportion, Th17/Treg ratio and Foxp3 mRNA expression level were significantly reduced. The above test results show progressive changes at different stages of the disease. Th17/Treg cells are abnormal when the thyroid function is normal in HT patients, but patients with subclinical hypothyroidism also have obvious changes. Among the three groups of hypothyroidism patients, subclinical hypothyroidism patients, and normal thyroid function patients, the first has the lowest proportion of Treg cells and the highest proportion of Th17 cells. As the disease worsens, the changes are most obvious in patients with hypothyroidism, indicating that the changes of Th17 and Treg cell subsets in HT patients are closely related to thyroid function. The imbalance of Th17/Treg cell ratio runs through different stages of HT, which is consistent with the results of HaiboXue and Pyzik [26, 
39]. Different thyroid functions may have some effects on Th17 and Treg cells [40], and the imbalance between them may play an important role in the pathogenesis of HT [41, 42]. It is speculated that the changes in the ratio of Th17 and Treg cells in HT patients at different stages may be related to changes in thyroid function and that whether it is directly related to immune response needs further investigation.

Th17/Treg cells participate in the immune damage of thyroid tissue itself through the abnormal immune response of T cells mediated by TGF- $\beta$ and other regulatory factors [43]. Treg cells mainly exert their biological effects by secreting TGF- $\beta$ and IL-10 [44]. TGF- $\beta$, as the main cytokine secreted by Treg cells, is always maintained at a low level during the pathogenesis of HT. The development of Treg cells and the expression of the key transcription factor Foxp3 depend on the existence of TGF- $\beta$, while the development of Th17 cells does not depend on TGF- $\beta$ [45]. In the TGF- $\beta 1$ deficient mouse model, it is confirmed that TGF- $\beta 1$ plays an important role in maintaining Foxp3 expressed by peripheral $\mathrm{CD} 4{ }^{+} \mathrm{CD} 25^{+}$Treg and exerting immunosuppressive effects. High concentration of TGF- $\beta 1$ can up-regulate the expression of Foxp3, induce the conversion of $\mathrm{CD} 4^{+} \mathrm{CD} 25^{-}$Treg into $\mathrm{CD} 4^{+} \mathrm{CD} 25^{+}$Treg and express Foxp3, which exerts a function similar to the naturally developed Treg cells in the thymus. Our results showed that the proportion of Th17 cells in PBMC, serum IL-17 and ROR- $\gamma$ t of HT patients were significantly increased, while the proportion of Treg cells and the mRNA level of Foxp3 were significantly reduced, and serum TGF- $\beta$ was reduced. We speculated that due to the decrease of Treg cells, the ability to suppress effector $\mathrm{T}$ cells is reduced. The body appears immune disorders leading to the disappearance of immune tolerance, the destruction of immune homeostasis, the activation of immune function, the activation of autoantibodies, and the induction of AID. Foxp3 could directly bind to ROR- $\gamma$ t to inhibit the transcription of IL-17 mRNA, thereby affected the function of Th17 cells. Foxp3 can directly bind to ROR- $\gamma$ t to inhibit the transcription of IL-17A mRNA, thereby affecting the function of Th17 cells. At the same time, the low level of Foxp3 attenuates the inhibition of ROR- $\gamma \mathrm{t}$ expression, cannot effectively promote the development of Treg cells, does not respond to TGF- $\beta$, and promotes Th17 cells to escape the immunosuppressive effect of Treg cells. The above-mentioned multiple factors are jointly involved in the imbalance of Th17/Treg cell subsets in HT patients [46]. And Th17/Treg are contribute to the pathopoiesis of Hashimoto's thyroiditis [47].

Our research results show that TPOAb and TgAb titers in HT patients increase with the progression of the disease and the increase in urinary iodine levels, and urinary iodine levels are positively correlated with TPOAb and TgAbtiters. This is consistent with the view that thyroid autoantibodies can aggravate immune damage [48]. The above point of view shows that high iodine may be the main environmental factor to induce the occurrence and development of HT. Excessive iodine intake can not only promote the occurrence of thyroid autoimmune reactions, but also further accelerate the occurrence of hypothyroidism in patients with abnormal thyroid autoimmunity. Maybe high iodine can aggravate the organic barrier of iodine, reduce the synthesis of thyroid hormone, and promote the occurrence of AIT in people with autoimmune background. In addition, excessive iodine causes thyroid tissue damage, accelerates the exposure of thyroid antigens, activates the autoimmune response, and induces the occurrence and development of HT [49]. About 90\% of HT patients have high titers of $\mathrm{TgAb}$ [50], 95\% of HT patients are TPOAb positive, and have a good correlation with thyroid auto reactive lymphocyte infiltration, but there are also a few healthy people who are TPOAbpositive. Approximately 4.3\% of TPOAb-positive subclinical hypothyroidism patients develop into clinical hypothyroidism every year, while only $2.6 \%$ of TPOAb-negative subclinical hypothyroidism patients develop into clinical hypothyroidism [51]. Th17 cells percentage were correlated with serum TPOAb, TgAb, and thyrotropin (TSH) levels in HT patients [52]. Our research results also show that the ratio of Th17/Treg cells is significantly positively correlated with TPOAb and TgAb titers, and the antibody titers of TPOAb and TgAb are highly correlated with Th17/Treg, IL-17 and ROR- $\gamma$ t. TPOAb and $\mathrm{TgAb}$ are thyroid auto-specific antibodies related to HT. $\mathrm{TPOAb}$ is the main antibody for antibody-dependent cell-mediated cytotoxicity. On the basis of T cell-mediated cell damage, it damages thyroid follicular epithelial cells, and finally leads to Hypothyroidism [53]. Th17/Treg imbalance mayplay a role in the production of thyroid autoantibodies and the abnormal immune response of $\mathrm{T}$ cells, participating in the immune damage of thyroid tissue itself. However, the interaction and mechanism of different iodine nutrition status, thyroid function, autoantibodies, Th17/Treg cells and their factors in the pathogenesis of HT should be studied in depth.

\section{Summary}

Th17 and Treg cells have mutually antagonistic effects. Th17/Treg balance can maintain the body in a steady state of immune tolerance. In inflammatory reactions or autoimmune diseases, the differentiation of $\mathrm{T}$ cells is affected, Th17 cell differentiation is enhanced, Treg cell differentiation is inhibited, and Th17/Treg cell balance is disrupted, which leads to body damage [54]. In thepast, it was believed that TPOAb and TgAb can damage thyroid cells through antibody dependent cell cytotoxicity (ADCC) or complement dependent cytotoxicity (CDC). Eventually it lead to hypothyroidism in patients with HT, so both cellular immunity and humoral immunity are involved in the pathogenesis of HT [55]. Our research results show that thyroid function changes in HT patients with different iodine nutritional status, which are often accompanied by significant increases in TPOAb and $\mathrm{TgAb}$ titers and are related to Th17/Treg cells and their cytokines and transcription factors. It is confirmed that the immune response of HT is mainly mediated by Th17 cells, accompanied by the production of autoantibodies, indicating that both the humoral and cellular immunity of HT patients are abnormal. However, it is not clear who plays the main role of humoral immunity or cellular 
immunity. Large-scale prospective studies are needed. The mutual influence and mechanism of Th17/Treg cells and their factors in the pathogenesis of HT need to be further explored.

\section{Conclusion}

The results of this study showed that the proportion of Th17 cells, Th17/Treg ratio, serum IL-17, TGF- $\beta$ and ROR- $\gamma$ t in HT patients were higher than those in the NC group, and gradually increased from HT-A to HT-C with statistical difference $(\mathrm{P}<0.05$ or $\mathrm{P}<0.01)$. The proportion of Treg cells and the level of Foxp3 were significantly lower than those of the control group, gradually decreasing from HT-A to HT-C. There were statistically significant differences between HT-C, HT-B and HT-A groups $(\mathrm{P}<0.05)$. Th17 cells and Th17/Treg ratio were positively correlated with TGF- $\beta$ and ROR- $\gamma \mathrm{t}(\mathrm{P}<0.05)$, and negatively correlated with Foxp3 $(\mathrm{P}<0.05)$. The ratio of Treg cells was negatively correlated with Th17 cell ratio, Th17/Treg ratio, TGF- $\beta$, ROR- $\gamma \mathrm{t}(\mathrm{P}<0.05)$, and positively correlated with Foxp3. In HT patients with different iodine nutritional status, the changes of Th17 and Tregs cells and their cytokines and transcription factors are same as the overall trend of HT patients basically. Among them, the proportion of Th17 cells, Th17/Treg ratio and IL-17 concen-tration increase, and the proportion of Treg cells decreased significantly, but the differences between the groups were not statistically significant. The proportion of Tregs and Foxp3 levels in the low TPOAb and TgAb groups were higher than those in the high TPOAb and TgAb groups. The rest showed a trend that the proportion of Th17 and Tregs cells and their cytokines and transcription factors in the high TPOAb and TgAb level group were higher than those in the low TPOAb and TgAb level group. Th17/Treg, IL-17 and ROR- $\gamma \mathrm{t}$ are more obvious, but the difference is not statistically significant. Urinary iodine levels in HT patients are positively correlated with TPOAb and TgAb. Antibody titers increase with disease progression and urinary iodine levels. The ratio of Th17/Treg cells change dynamically in different degrees of HT and are positively correlated with thyroid autoantibodies. This indicates that the imbalance of Th17/Treg cell subsets may be involved in the autoimmune damage of HT. In particular, urinary iodine, TPOAb and TgAb antibody titers have a greater correlation with Th17/Treg, IL-17 and ROR- $\gamma \mathrm{t}$, but the mechanism of action is unclear in the pathogenesis of HT. The results of this study and other studies have confirmed that abnormalities in urine iodine levels, thyroid function, TPOAb, TgAb and Th17/Treg cells and related factors are jointly involved in the occurrence and development of HT. In-depth research on the interaction between different iodine nutritional status, thyroid function stratification, thyroid autoantibodies and Th17/Treg cells and related factors and their mechanism of action will open up new ways for the precise prevention and treatment of HT. However, the results of the above indicators in HT patients are not the same, which may be related to the geographical distribution, number of specimens, gender differences, disease composition ratio, severity, and stage. Therefore, it is necessary to carry out multi-center and large-sample prospective studies to reduce the error of results and provide scientific basis for accurate diagnosis, treatment and prevention of HT.

\section{Acknowledgements}

Cangzhou Key R \& D Plan and Guidance Project (Project No: 1833020011).

Natural Science Foundation of Cangzhou Medical College (No. 18Z015).

\section{References}

[1] Xiang Guangda. Clinical Thyroidology [M]. People's Medical Publishing House, 2013: 200.

[2] Hu S, Rayman MP. Multiple Nutritional Factors and the Risk of Hashimoto's Thyroiditis [J]. Thyroid. 2017, 27 (5): 597-610.

[3] Li HX, Xiang N, Hu WK, et al. Relation between therapy options for Graves' disease and the course of Graves' ophthalmopathy: a systematic review and met analysis $[\mathrm{J}]$. $\mathrm{J}$ Endocrinol Invest, 2016, 39 (11): 1225-1233.

[4] Hou ZJ, Mu ZX, Wang CC. Research Progress of Th17/Treg Cells and Their Transcription Factors in Autoimmune Diseases [J]. American Journal of Clinical and Experimental Medicine 2019, 7 (4): 83-92.

[5] Chinese Medical Association Endocrine Credits "China thyroid disease treatment guidelines" Writing Group. China thyroid disease treatment guidelines-Thyroiditis [J]. Chin J inter Med, 2 008, 47 (9): 784-788.

[6] Chinese Medical Association Endocrine Credits "China thyroid disease treatment guidelines" Writing Group. China thyroid disease treatment guidelines-iodine deficiency disorders $[\mathrm{J}]$. Journal of Internal Medicine, 2008, 47 (8): 689-690.

[7] Huang J, Yu PF, Yang ZP, Clinical application of thyroid stimulating hormone and thyroid autoantibodies in the diagnosis of thyroid diseases [J]. Chinese Journal of Control of Endemic Diseases, 2015, 30 (1): 65-66.

[8] Wang B, Meng JH. Research of thyroid autoantibodies in the diagnosis of Graves' Disease and Hashimoto's Thyroiditis [J]. Chin J Lab Diagn, 2018, 22 (2): 207-209.

[9] Dong X. The diagnostic value of serum thyroid autoantibodies for autoimmune thyroid disease [J]. ClinMed, 2018, 38 (5): 40-41.

[10] Yang J, Li Q. Clinical application of thyroid stimulating hormone and thyroid autoantibodies in the diagnosis of thyroid diseases [J]. ProceClin Med, 2018, 27 (6): 424-426.

[11] Zou GH, Sun FY, Dai JQ, et al. Role of follicular regulatory T cells in the pathogenesis of Hashimoto thyroiditis [J]. Lab Med, 2019, 34 (8): 682-686.

[12] Wang YG, Yan SL, Zhao SH, et al. Correlation of Hashimoto's thyroiditis with urine iodine level among the coastal districts in Shandong Province [J]. Chin J Endoc \& Meta, 2004, 20 (4): 337-338. 
[13] Ma QQ, Liang QH, Sun L, et al. Expression and significance of $\mathrm{CD}^{+} \mathrm{CD}^{+} 5 \mathrm{RO}^{+}$memory $\mathrm{T}$ cell in peripheral blood of patients with Hashimoto's thyroiditis [J]. Chinese Journal of Immunology, 2016, 32 (10): 1527-1531.

[14] Chen YM, Hu FQ, Huang H, et al. The effect of Treg and Th17 cells in Hashimoto's thyroiditis patiens with normal thyroid function [J] Med J West China, 2018, 30 (10): 1438-1442.

[15] Chen YX. Correlation between TNF- $\alpha$ and thyroid autoantibodies in hashimoto thyroiditis patients with different iodine intake [D]. Hebei Medical University, 2011.

[16] Kleinewietfeld M, HaflerDA. The plasticity of human Treg and Th17 cells and its role in autoimmunity [J]. Semin Immunol. 2013, 25 (4): 305-312.

[17] Singh B, Schwartz JA, Sandroc kC, et al. Modulation of autoimmune diseases by interleukin (IL)-17 producing regulatory T helper (Th17) cells [J]. Indian J Med Res, 2013, 138 (5): 591-594.

[18] Figueroa-Vega N, Alfonso-Pérez M, Benedicto I, et al. Increased circulating pro-inflammatory cytokines and Th17 lymphocytes in Hashimoto's thyroiditis [J]. J Clin Endocrinol Metab, 2010, 95, (2): 953-962.

[19] Shi Y, Wang H, Su Z, et al. Differentiation imbalance of Th1/Th17 in peripheral blood mononuclear cells might contribute to pathogenesis of Hashimoto's thyroiditis [J]. Scand J Immunol. 2010, 72 (3): 250-255.

[20] Wang S, Baidoo SE, Liu Y, et al. T cell-derived leptin contributes to increased frequency of $\mathrm{T}$ helper type 17 cells in female patients with Hashimoto's thyroiditis [J]. Clin Exp Immunol. 2013, 171 (1): 63-68.

[21] Xie XY, Xi' er NY, Guo T, et al. The Alteration of the Th1, Th2, Th17, Treg Cells in the Peripheral Blood in Patients with Grave's Disease (GD) and Hashimoto's Thyroiditis with Hypothyroidism. J Radioimmunology, 2011, 24 (3): 290-293.

[22] Chen WZ. Immune imbalance of Th17/Treg in hashimoto's thyroiditis [D]. Nanchang Unive, 2012.

[23] Chen ZJ, LIU C, LI Q, et al. The changes and significance of Th17 cells and related cytokines in autoimmune thyroid diseases [J]. Immunological Journal, 2011, 27 (9): 785-788.

[24] Zhao JY. Detection and significance of Th17 cells in hashimoto's thyroiditis and Graves' disease [D]. Master's Thesis of Jilin University, 2012.

[25] Sun QK, Chen ZL, LI CH, et al. The signaling abnormalities of IL-6/TGF- $\beta$ in Th17/Treg cell imbalance in Hashimoto's thyroiditis $[\mathrm{J}]$. Chinese Journal of Endocrinology and Metabolism, 2015, 31 (4): 320-326.

[26] Xue HB, Ma L, LI YB, et al. Correlation between Treg/Th17 cell and autoimmunity of Hashimoto's thyroiditis [J]. China Journal of Modern Medicine, 2012, 22 (23): 67-71.

[27] Jin X, Shang XM, Chen HB, et al. Study on dynamic changes and significance of the peripheral blood regulatory $\mathrm{T}$ cells (Treg) and Th17 cells in patients with Hashimoto's thyroiditis [J]. J ClinExper Med, 2018, 17 (19): 2092-2094.

[28] Ceyla Konca Degertekin, Banu Aktas Yilmaz, Fusun Balos Toruner, et al. Circulating Th17 cytokine levels are altered in Hashimoto's thyroiditis [J], Cytokine 80 (2016) 13-17.

[29] Marazuela M, García-López MA, Figueroa-Vega N, et al.
Regulatory T cells in human autoimmune thyroid disease [J]. J Clin Endocrinol Metab. 2006, 91 (9): 3639-3646.

[30] Gao ST, Zhu TN, Zhang YN, et al. The frequency and function of $\mathrm{CD} 4{ }^{+} \mathrm{CD} 25^{+}$regulatory $\mathrm{T}$ cells in autoimmune thyroid diseases Chin J Immu, 2011, 27 (3): 269-273.

[31] Mao C, Wang S, Xiao Y, et al. Impairment of regulatory capacity of $\mathrm{CD} 4{ }^{+} \mathrm{CD} 25^{+}$regulatory $\mathrm{T}$ cells mediated by dendritic cell polarization and hyperthyroidism in Graves' disease [J]. J Immunol. 2011, 186 (8): 4734-4743.

[32] Zheng LT, Yu SP, Wang N, et al. Regulatory T cells and TGF- $\beta$ in autoimmune Therole of thyroid disease [J]. Shandong Med 2012, 52 (01): 81-82.

[33] Yang ZF, Wu F, TANG JZ, et al. Expression of regulatory T cells in patients with hashimoto's thyroiditis and its significance [J]. China General Practice, 2013, 16 (7C): 2477-2479.

[34] Bi JH, Hao LX, Huang F, et al. The detection and clinical significance of $\mathrm{CD}^{+} \mathrm{CD} 25^{+} \mathrm{Foxp} 3^{+}$regulatory $\mathrm{T}$ cells and IL-10 in peripheral blood of hashimoto thyroiditis patients [J]. Journal of clinical and experimental medicine, 2017, 16 (1): $38-40$.

[35] Glick AB, Wodzinski A, Fu P, et al. Impairment of regulatory $\mathrm{T}$ cell function in autoimmune thyroid disease [J]. Thyroid, 2013, 23 (7): 871-878.

[36] Bao QY, Xu YS, Yang HY. Regulatory T Cells and Endocrine Diseases. Med Reca, 2014, 20 (18): 3278-3280.

[37] Zhu JF, Yamane H, Paul WE. Differentiation of effector CD4 T cell populations [J]. Annu Rev Immunol. 2010; 28: 445-489.

[38] Kleinewietfeld M, Hafler DA. The plasticity of human Treg and Th17 cells and its role in autoimmunity [J]. Semin Immunol. 2013, 25 (4): 305-312.

[39] Pyzik A, Grywalska E, Matyjaszek-Matuszek B, et al. Immune disorders in Hashimoto's thyroiditis: what do we know so far? [J]. J Immunol Res. 2015, 2015: 979167.

[40] Rodien P, Madec AM, Ruf J, et al. Antibody-dependent cell-mediated cytotoxicity in autoimmune thyroid disease: relationship to antithyroperoxidase antibodies $[\mathrm{J}]$. J ClinEndocrinol Metab. 1996, 81 (7): 2595-2600.

[41] Xue HB, Yu XR, Ma L, et al. The possible role of $\mathrm{CD} 4{ }^{+} \mathrm{CD} 25^{\text {high }} \mathrm{Foxp}^{+} / \mathrm{CD}^{+}{ }^{+} \mathrm{L}-17 \mathrm{~A}^{+}$cell imbalance in the autoimmunity of patients with Hashimoto thyroiditis [J]. Endocrine. 2015, 50 (3): 665-673.

[42] González-Amaro R, Marazuela M. T regulatory (Treg) and T helper17 (Th17) lymphocytes in thyroid autoimmunity [J]. Endocrine. 2016, 52 (1): 30-38.

[43] Li C, Ebert PJ, Li QJ. T cell receptor (TCR) and transforming growth factor $\beta$ (TGF- $\beta$ ) signaling converge on DNA (cytosine-5)-methyltransferase to control forkhead box protein 3 (foxp3) locus methylation and inducible regulatory $\mathrm{T}$ cell differentiation [J]. J Biol Chem. 2013, 288 (26): 19127-19139.

[44] Cai H, Kong W, Zhou T, et al. Radiofrequency ablation versus reresection in treating recurrent hepatocellular carcinoma: a meta-analysis [J]. Medicine, 2014, 93 (22): e122.

[45] Hirota K, Martin B, Veldhoen M. Development, regulation and functional capacities of Th17 cells [J]. Sem in Immunopathol, 2010, 32 (1): 3-16. 
[46] Yoshimura A, Muto G. TGF- $\beta$ function in immune suppression [J]. Curr Top MicrobiolImmunol, 2011, 350: 127-147.

[47] Liu YZ, Tang XY, Tian J, et al. Th17/Treg Cells Imbalance and GITRL Profile in Patients with Hashimoto's Thyroiditis [J]. International Journal ofMolecular Sciences, 2014, 15, 21674-21686.

[48] Caturegli P, De Remigis A, Chuang K, et al. Hashimoto'sthyroiditis: celebrating the centennialthrough the lens of the Johns Hopkins hospital surgical pathology records [J]. Thyroid, 2013, 23 (2): 142-150.,

[49] Latrofa F, Fiore E, Rago T, et al. Iodine contributes to thyroid autoimmunity in humans by unmasking a cryptic epitope on thyroglobulin [J]. J Clin Endocrinol Metab, 2013, 98 (11): E1768-1774.

[50] Liu M, Zhao L, Gao Y, et al. Epitope recognition patterns of thyroglobulin antibody in sera from patients with Hashimoto's thyroiditis on different thyroid functional status [J]. ClinExp Immunol, 2012, 170 (3): 283-290.
[51] Antonelli A, Ferrari SM, Corrado A, et al. Autoimmune thyroid disorders [J]. Autoimmun Rev, 2015, 14 (2): 174-180.

[52] Haibo Xue, Yuhua Yang, Ying Zhang, et al. Macrophage Migration Inhibitory Factor Interacting withTh17 Cells May Be Involved in the Pathogenesis of Autoimmune Damage in Hashimoto's Thyroiditis [J]. Mediators of Inflammation 2015 (2): $1-9$

[53] Rieu M, Richard A, Rosilio M, et al. Effects of thyroid status on thyroid autoimmunity expression in euthyroid and hypothyroid patients with Hashimoto's thyroiditis [J]. ClinEndocrinol. 1994, 40 (4): 529-35.

[54] Uqba Khan, Ghazanfar H. T. Lymphocytes and Autoimmunity [J]. Int Rev Cell Mol Biol. 2018, 341: 125-168.

[55] Gao Y, LIU MM, XIU RJ. Advances in the immunological pathogenesis of hashimoto's thyroiditis [J]. Journal of Internal Medicine Concepts \& Practice, 2013, 8 (6): 392-396. 